\title{
A pilot study of the endomicroscopic assessment of tumor extension in Barrett's esophagus-associated neoplasia before endoscopic resection
}

Authors

Institutions
Werner Dolak ${ }^{1}$, Ildiko Mesteri², Reza Asari ${ }^{3}$, Matthias Preusser ${ }^{4}$, Barbara Tribl ${ }^{1}$, Friedrich Wrba ${ }^{2}$, Sebastian F. Schoppmann ${ }^{3}$, Michael Hejna ${ }^{4}$, Michael Trauner' ${ }^{1}$, Michael Häfner'1, Andreas Püspök ${ }^{1}$

Institutions are listed at the end of article. submitted 30. April 2014 accepted after revision 15. July 2014

\section{Bibliography}

Dol http://dx.doi.org/ 10.1055/s-0034-1377935

Published online: 24.10 .2014

Endoscopy International Open 2015; 02: E19-E28

(c) Georg Thieme Verlag KG Stuttgart . New York E-ISSN 2196-9736

\section{Corresponding author \\ Werner Dolak, MD}

Division of Gastroenterology

and Hepatology

Department of Internal

Medicine III

Medical University of Vienna

Waehringer Guertel 18-20

1090 Vienna

Austria

Fax: +43140400 47350 werner.dolak@meduniwien.ac. at
Background and study aims: Barrett's esophagus (BE)-associated neoplasia can be treated endoscopically, but accurate assessment before intervention is challenging. This study aimed to investigate the role of confocal laser endomicroscopy (CLE) as an adjunct in the endoscopic treatment of BE-associated neoplasia by assessing lateral tumor and subsquamous tumor (SST) extension. Patients and methods: In the context of a prospective, single-arm pilot clinical trial, patients referred for endoscopic resection of BE-associated neoplasia (high grade dysplasia and esophageal adenocarcinoma) underwent high definition, white light endoscopy with narrow-band imaging (NBI). Then, CLE mapping of suspected neoplastic lesions was performed by another endoscopist, partially blinded to the previous findings, before the patients underwent endoscopic mucosal resection (EMR) or endoscopic submucosal dissection (ESD), depending on lesion size and anticipated histology.

\section{Introduction \\ $\nabla$}

The incidence of esophageal adenocarcinoma (EAC) is rapidly increasing in the Western world $[1,2]$. Barrett's esophagus (BE) is the most important premalignant condition in the development of EAC, with a known risk of malignant transformation of $0.12 \%$ to $0.4 \%$ per year $[3,4]$. Despite technologic improvements in endoscopic imaging, the detection and precise delineation of neoplastic lesions in BE remain difficult. This challenge is further increased by subsquamous tumor (SST) extension, defined as the presence of intestinal metaplasia or related neoplasia underneath the surrounding squamous epithelium [5]. Recent analyses of resected specimens of Barrett's adenocarcinoma have revealed SST extension in a high number of cases [6,7]. Although the clinical impact of SST extension is still controversial, because its diagnostic accessibility is lim-
Results: In 7 of 38 patients (18\%), CLE revealed additional neoplastic tissue compared with prior white light endoscopy and NBI: 2 concomitant lesions, 2 cases of lateral tumor extension within the Barrett's epithelium, and 3 cases of previously undetected SST extension. Overall, en bloc resection (tumor-free lateral margin) was achieved in 28 of 34 neoplastic lesions (82\%), and complete resection (tumor-free lateral and basal margins) in 21 of 34 neoplastic lesions (62\%).

Conclusions: CLE-assisted endoscopic resection of BE-associated neoplasia was safe and effective in this study, as proved by a high additional diagnostic yield of CLE (including visualization of occult SST extension) and a favorable rate of en bloc resection. The clinical value of CLE for assisting endoscopic therapy of BE-associated neoplasia deserves further evaluation in randomized controlled trials.

ited, it interferes with the adequate pre-interventional planning of endoscopic resection, especially in patients with tumors arising near the squamocolumnar junction. A diagnostic modality that might improve the visualization of lateral tumor extension and SST extension in BE-associated neoplasia is confocal laser endomicroscopy (CLE), which allows the microscopic scanning of focal points below the surface of the gastrointestinal tract by means of fluorescence excitation with laser light [8]. Initial studies on the use of CLE in patients with $\mathrm{BE}$ have shown high rates of sensitivity and specificity in the detection of BE and BEassociated neoplastic changes $[9,10]$. This study aimed to investigate the diagnostic benefit of CLE for the assessment of lateral tumor extension and SST extension in BE-associated neoplasia before endoscopic resection, and the impact of CLE-assisted endoscopic resection on patients' clinical outcomes. 
Table 1 Summary of patient characteristics.

\begin{tabular}{|c|c|c|}
\hline & No. & Percentage \\
\hline Gender, M:F & $32: 6$ & $84 \%: 16 \%$ \\
\hline Median age, y (range) & $69(43-84)$ & NA \\
\hline BE surveillance program, Y:N & $14: 24$ & $37 \%: 63 \%$ \\
\hline Histology at referral, EAC:HGD ${ }^{1}$ & $26: 12$ & $68 \%: 32 \%$ \\
\hline $\begin{array}{l}\text { EUS staging of EAC (uT1m: } \\
\text { uT1sm) }\end{array}$ & $23: 3$ & $88 \%: 12 \%$ \\
\hline Median BE length (range) & $\begin{array}{l}\text { C1M3 } \\
\text { (C0M1 - } \\
\text { C11M12) }\end{array}$ & NA \\
\hline ER type per lesion, EMR:ESD² & 25:11 & $69 \%: 31 \%$ \\
\hline High risk after $\mathrm{ER}, \mathrm{Y}: \mathrm{N}^{3}$ & $15: 19$ & $44 \%: 56 \%$ \\
\hline $\begin{array}{l}\text { Surgical therapy within follow-up, } \\
Y: N\end{array}$ & $11: 27$ & $29 \%: 71 \%$ \\
\hline
\end{tabular}

BE, Barrett's esophagus; EAC, esophageal adenocarcinoma; HGD, high grade dysplasia; EUS, endosonography; ER, endoscopic resection; EMR, endoscopic mucosal resection; ESD, endoscopic submucosal dissection.

Upgrade of two HGDs by board-certified pathologist already considered.

2 Including one unsuccessful ESD.

${ }^{3}$ Study patients without detectable lesions not considered.

\section{Patients and methods}

$\nabla$

This prospective, single-arm pilot clinical trial was conducted at a university-based center in Austria. The study protocol was approved by the internal review board of the Medical University of Vienna (EK 697/2009) and was registered at clinicaltrials.gov (NCT01124994). Patients who were referred for the endoscopic resection of high grade dysplasia (HGD) or EAC arising in $\mathrm{BE}$ were screened for inclusion in this study. HGD at referral had to be confirmed by a reference pathologist at our institution. Patients with EAC had to undergo endoscopic ultrasonographic (EUS) staging to exclude an advanced local tumor stage or regional lymph node metastases. Other exclusion criteria in this trial were general contraindications to endoscopy (e.g., recent myocardial infarction) or endoscopic resection (e.g., impaired coagulation status), a known allergy to fluorescein (the fluorescent dye used for CLE), and age younger than 18 years. Informed consent was obtained in written form from patients without any of the exclusion criteria, and they were enrolled in the study. All study procedures were done by four experienced endoscopists with a high level of expertise in the respective endoscopic modality. Midazolam and/or propofol was used for conscious sedation during the following three phases of each study procedure: (1) lesion assessment with high definition, white light endoscopy and narrow-band imaging (NBI), (2) lesion assessment with CLE, and (3) endoscopic resection. SPSS version 19.0 was used to process all data in the context of this study.

Lesion assessment by high definition, white light endoscopy and narrow-band imaging

At the beginning of each study procedure, upper gastrointestinal endoscopy with a standard high definition, white light gastroscope (GIF-H180 with Evis Exera II processor; Olympus America, Center Valley, Pennsylvania, USA) was performed to identify the location and macroscopic extension of BE-associated neoplasia. NBI was used to screen for irregular vascular patterns. Dye spraying (e.g., with acetic acid) was not allowed at this point of the examination in order to avoid visual interference with the subsequent CLE. In patients who had a short BE segment and did not have a large hiatal hernia, a transparent hood was attached to the tip of the endoscope to stabilize its position in the distal esophagus during inspection of the mucosa. Areas with macroscopic features suspicious for neoplasia were numbered consecutively, documented by picture capturing, and marked circumferentially on the pictures by the endoscopist who performed the examination.

\section{Lesion assessment by confocal laser endomicroscopy}

Directly after high definition, white light endoscopy, the study procedure was continued with a scope-based confocal laser endomicroscope (EG-3870CIFK with ISC-1000 confocal endomicroscopy processor; Pentax, Tokyo, Japan, and Optiscan Pty. Ltd., Notting Hill, Victoria, Australia). A total of $5 \mathrm{~mL}$ of a $10 \%$ solution of fluorescein sodium was administered intravenously to enhance tissue fluorescence for CLE. The lesions identified by high definition, white light endoscopy were mapped with CLE by another endoscopist, who was informed about the number and location of the lesions but blinded to their previously assessed extension. CLE mapping was done by obtaining "optical biopsies" (scans from the surface to maximal depth in the tissue) at the center of each lesion, then centrifugally at each point of the compass in 5-mm intervals until there was no suspicion of neoplasia on CLE. For lesions adjacent to the squamocolumnar junction, CLE scanning was performed in the same way, with special focus on obtaining deeper CLE pictures underneath the squamous epithelium to detect SST extension. Finally, CLE scanning was done circularly along the demarcated boarders of each lesion, which were then marked by argon plasma coagulation with a VIO $200 \mathrm{D}$ processor (Erbe Elektromedizin GmbH, Tübingen, Germany). As with high definition, white light endoscopy, the final lesion size assessed by CLE was documented by picture capturing. At additional areas of BE without prior macroscopic suspicion of dysplasia, CLE pictures were obtained, and corresponding conventional biopsy specimens were taken with a large-capacity forceps according to the Seattle protocol to provide dysplasia-negative controls [11].

\section{Endoscopic resection}

Because of the special characteristics of the CLE scope (large diameter, rigid tip), it was necessary to switch back to a standard gastroscope for the final part of the study procedure, endoscopic resection. Before endoscopic resection and after the topical application of acetic acid, the entire BE segment was observed again by both endoscopists involved in the previous lesion assessment. Depending on the size and suspected histology of the lesions marked by argon plasma coagulation, the following resection techniques were used: multiband ligation (Duette; Cook Medical, Bloomington, Indiana, USA) or cap and snare (EMR Kit; Olympus) en bloc endoscopic mucosal resection (EMR) for lesions up to 15 $\mathrm{mm}$ in diameter, irrespective of the underlying histology; multiband ligation or cap-assisted piecemeal EMR for HGD lesions larger than $15 \mathrm{~mm}$; and endoscopic submucosal dissection (ESD) with a HybridKnife (I-type or O-type with VIO 200D processor; Erbe) for EAC lesions larger than $15 \mathrm{~mm}$.

\section{Processing of the resected specimens}

The fresh resection specimen was pinned onto a cork board. If a specimen could not be oriented after a piecemeal resection, multiple punch biopsies were taken to evaluate the resection margins. The tissue was fixed in formalin; after fixation, the basal and radial resection margins were inked, and the entire specimen was submitted in vertical serial cross sections and oriented during paraf- 


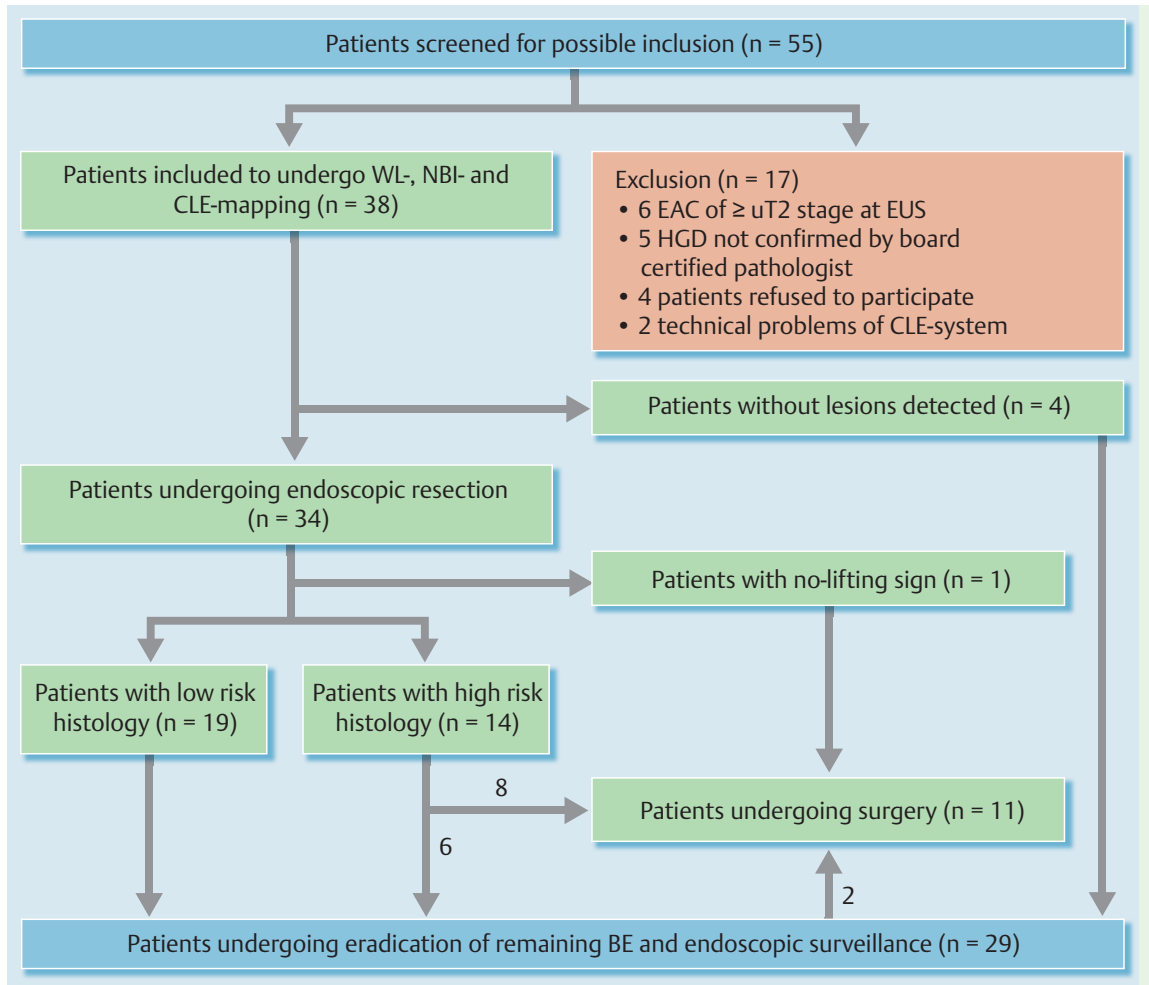

Fig. 1 Study flow chart. EAC, esophageal adenocarcinoma; EUS, endosonography; HGD, high grade dysplasia; CLE, confocal laser endomicroscopy; WL, white light; NBI, narrow-band imaging; BE, Barrett's esophagus.

fin embedding. The pathology report included the following information: grade of differentiation; depth of invasion, indicated as $\mathrm{m} 1$ (intraepithelial carcinoma), $\mathrm{m} 2$ (invasion of the lamina propria), $\mathrm{m} 3$ (invasion of the muscularis mucosae), $\mathrm{sm} 1$ (invasion of the shallowest one-third of the submucosa $[<500 \mu \mathrm{m}])$, or $\geq \mathrm{sm} 2$ (deeper submucosal invasion [> $500 \mu \mathrm{m}]$ ); evidence of vascular invasion; and assessment of the radial and deep resection margins $[12,13]$. A specimen with neoplasia-free lateral margins and a histologically confirmed lesion was considered to be an "en bloc" resection. If both the basal and lateral margins of the specimen were negative for neoplasia, the lesion was classified as "completely" resected. En bloc and complete resection rates were calculated for the entire study population as well as for subgroups according to the final histology.

\section{Follow-up}

Patients at high risk for recurrence or metastatic disease after the endoscopic treatment of EAC - that is, those with a poorly differentiated tumor (G3), tumor invasion below the upper third of the submucosa ( $\geq \mathrm{pT} 1 \mathrm{sm} 2$ ), lymphatic vessel invasion (L1), vascular invasion (V1), perineural invasion (Pn1), or tumor invasion of the basal or lateral resection margins (R1) - were evaluated for additional surgical treatment. Patients who were not at high risk or who should have undergone surgery but were unfit or refused were followed endoscopically. Patients underwent upper gastrointestinal endoscopy with biopsy assessment according to the Seattle protocol every 3 months during the first year after endoscopic resection, then annually [8]. Patients with remaining areas of $\mathrm{BE}$ after endoscopic resection underwent radiofrequency ablation (RFA), provided that the first endoscopic follow-up after resection of BE-associated neoplasia did not reveal any focal lesions or advanced neoplasia. RFA was repeated until no histologic evidence of BE was found.

\section{Results}

$\nabla$

Between June 2011 and May 2013, 55 patients were screened, of whom 38 (32 men, 6 women; median age, 69; range, 43-84) were eventually included in the study ( $\bullet$ Fig. 1 ; Tables 1,2). In $14(37 \%)$ of the 38 patients, BE-associated neoplasia had been diagnosed in the context of endoscopic surveillance for alreadyknown BE. Of the 38 patients, 3 (8\%) had already undergone fundoplication for the treatment of gastroesophageal reflux disease (GERD), and 3 ( $8 \%$ ) had already undergone endoscopic resection of HGD at least 2 years previously. The underlying pathology in the 38 study patients (after re-evaluation of all cases of HGD by the reference pathologist) included 26 cases of EAC and 12 cases of HGD. EUS staging showed a definite invasion of the submucosal layer in 3 of the 26 patients with EAC ( $\bullet$ Fig. 2 a).

High definition, white light endoscopy; narrow-band imaging; and confocal laser endomicroscopy mapping High definition, white light endoscopy and NBI mapping revealed a total of 34 lesions in 34 of the 38 patients with a median BE length of C1M3 (range, C0M1 - C11M12) according to the Prague classification [14]. SST extension was visible in 3 cases, consisting of a slightly elevated squamous epithelium with an irregular vascular architecture ( $\bullet$ Fig. $\mathbf{2}$ b). In 4 of the 38 patients, no circumscribed lesion could be detected by white light endoscopy or NBI. The mean lesion size based on macroscopic features was $14.1 \pm$ $5.7 \mathrm{~mm}$. CLE confirmed the macroscopic size in 29 of the 34 lesions (including the 3 cases of SST extension already known). In 5 of the 34 macroscopic lesions, CLE led to an extension of the endoscopically defined lesion size as a result of the detection of neoplastic tissue outside the macroscopically estimated boarders: in 2 cases lateral tumor extension within the BE and in 3 cases SST extension that had not been detected before. The increase in lesion size also led to a change of the resection technique (ESD instead of EMR) in 2 patients in whom CLE had detect- 


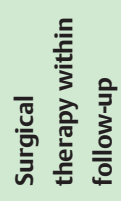

๗ั

兹

壱壱

๖

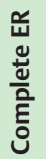

음

운

도 뜬

운

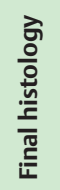

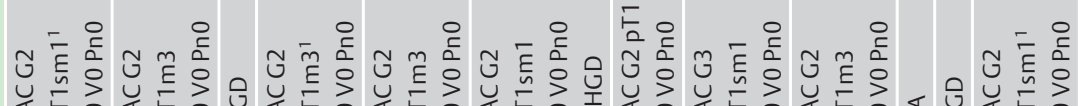

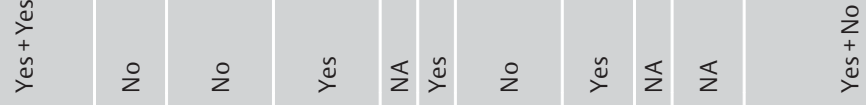

产

$\sum_{\dot{w}}^{\infty}$

$\sum_{i=1}^{\infty} \sum_{w}^{\infty}$

$\sum_{i=1}^{\infty} \quad \sum$

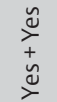

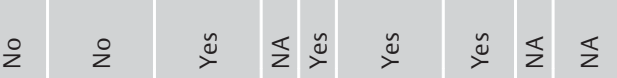

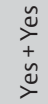

色

กับ

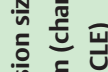

$\frac{1}{12}$

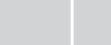

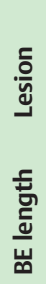

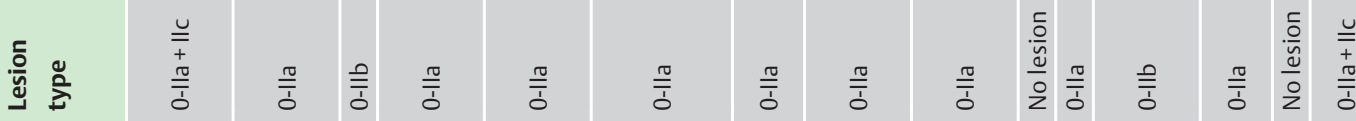

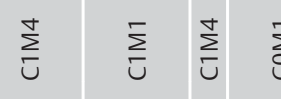

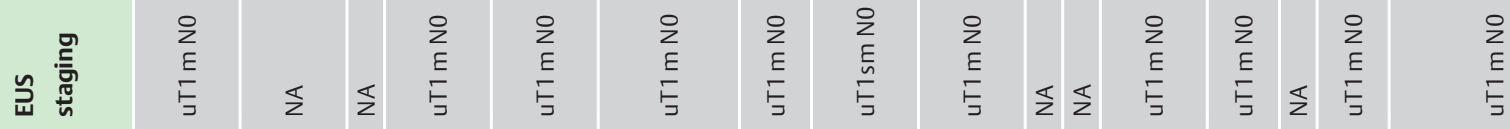

范密言

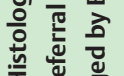

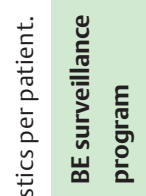

ড̣

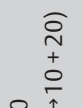

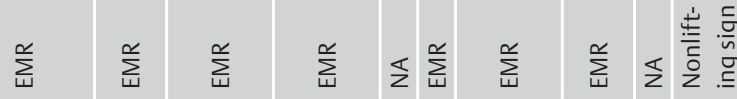

Tु

U⿺辶寸

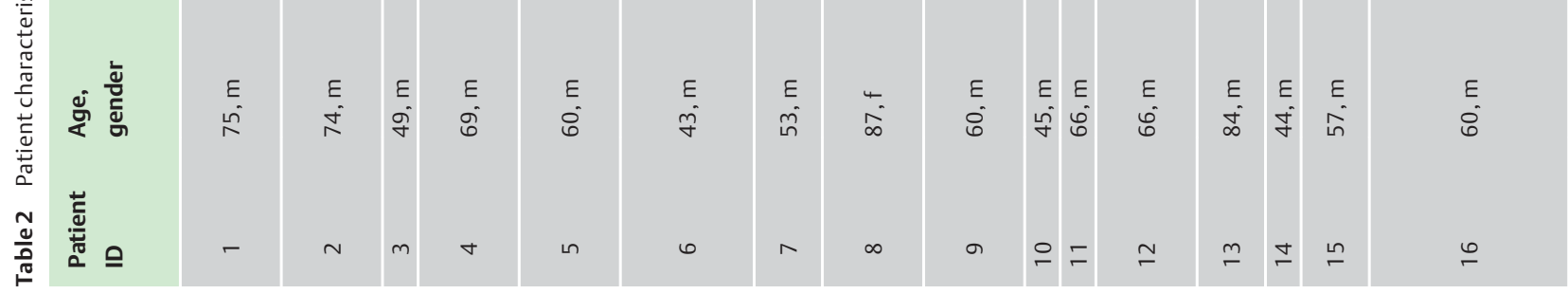




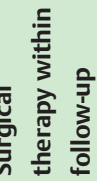

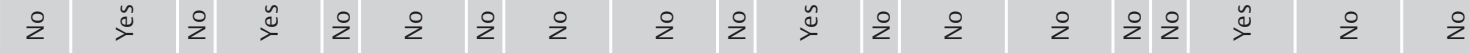

는 品

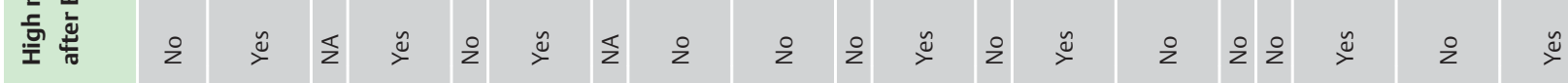

遶

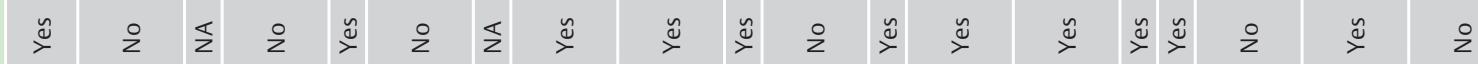

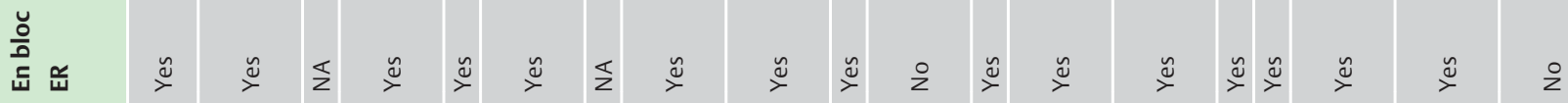

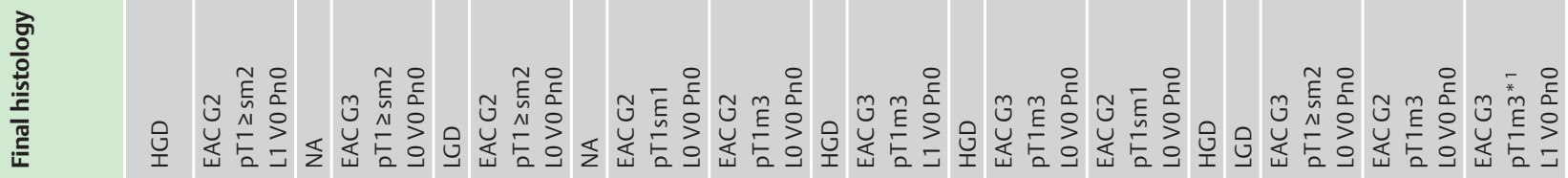

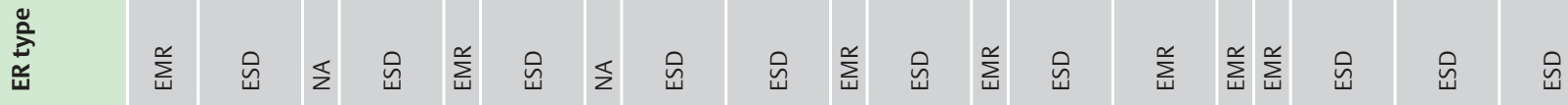

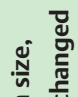

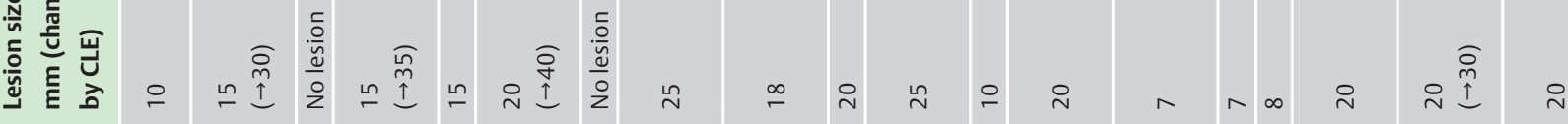

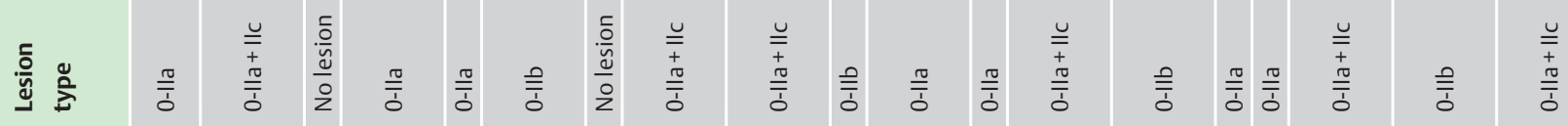

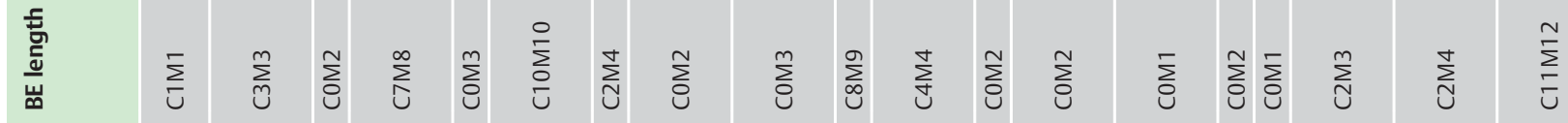

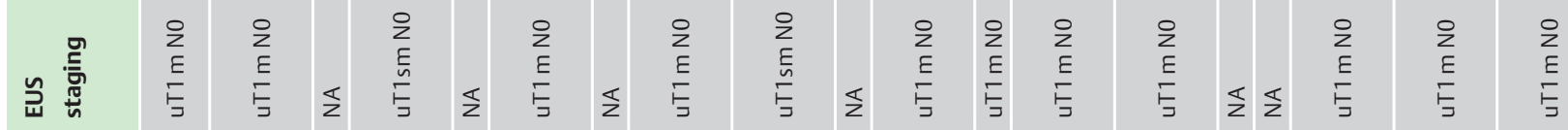

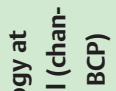

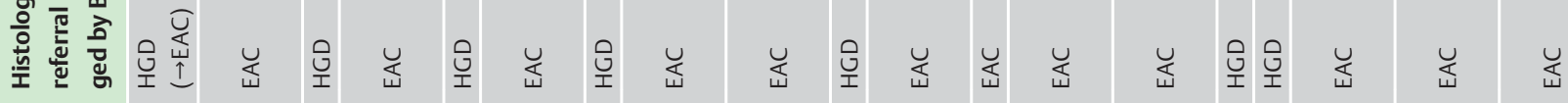
总

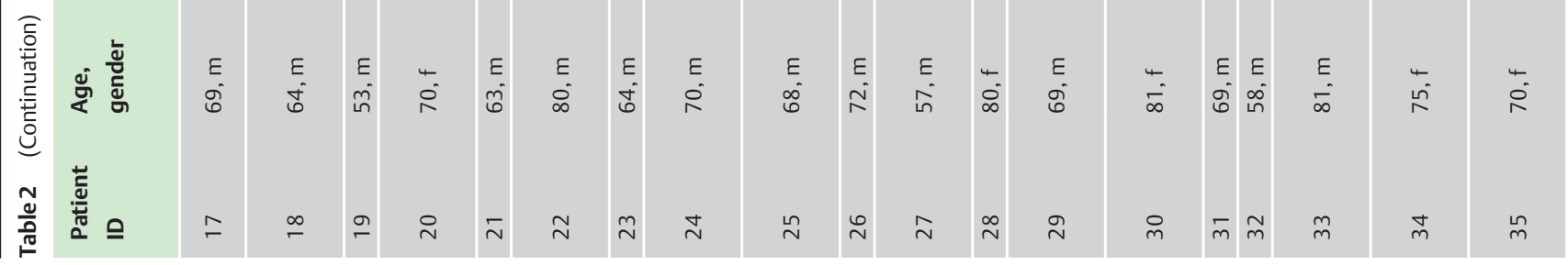


ed SST extension. Furthermore, CLE scanning of additional areas of $\mathrm{BE}$ without prior macroscopic suspicion of dysplasia and the adjacent squamous epithelium detected 2 concomitant lesions, 10 and $20 \mathrm{~mm}$ in size, that had not been detected by white light endoscopy and NBI. Histologically, one turned out to be HGD, and the other was an early squamous cell carcinoma. Therefore, CLE provided an incremental diagnostic yield in $7(18 \%)$ of the 38 patients. It detected a total of 36 lesions in 34 of the 38 patients, with a mean lesion size of $16.5 \pm 9.0 \mathrm{~mm}(\bullet \mathrm{Fig}$. 3). It failed to detect the expected HGD in those 4 of the 38 patients in whom the results of white light endoscopy and NBI had already been negative $(\bullet$ Fig. $2 \mathrm{c}$ ). The topical application of acetic acid before endoscopic resection neither showed any visual abnormalities at the areas with additional neoplastic tissue detected by CLE nor revealed any further lesions or lesion extensions.

\section{Endoscopic resection and histologic evaluation}

Endoscopic resection was performed for the 36 detected lesions (25 EMR, 11 ESD). One ESD could not be completed because of a nonlifting sign, suspicious for T2 tumor stage (later confirmed by surgery). All other endoscopic resections could be completed without technical issues. Bleeding in 13 (38\%) of 34 patients was the only interventional adverse event but could be managed by argon plasma coagulation and/or clipping in all cases.

Histologic evaluation of the resected specimens revealed 22 cases of EAC (half of them showing invasion of the submucosa), 8 cases of HGD, 3 cases of low grade dysplasia (LGD), 1 squamous cell carcinoma (in a patient with concomitant EAC), and $1 \mathrm{BE}$ without dysplasia ( $\bullet$ Fig.4). The 6 cases of SST extension detected previously were confirmed by histology. Of the 34 neoplastic lesions proven by histology, an en bloc resection was achieved in $28(82 \%)$ and a complete resection in 21 (62\%). These rates differed among the histologic subgroups $(\bullet$ Table 3). After CLE evaluation, 31 conventional biopsy specimens were taken from supposedly nondysplastic BE in 19 patients with BE extension larger than COM2. Histologically, 28 of the 31 biopsy specimens showed BE without dysplasia, and 3 of the 31 showed LGD.

The 4 patients without lesions detected by white light endoscopy, $\mathrm{NBI}$, or CLE presented with C0M2, COM2, C2M4, and C6M6 BE. To avoid overtreatment, only the 2 patients with noncircular BE underwent endoscopic resection by EMR. The others underwent another biopsy according to the Seattle protocol and RFA thereafter. Both the resected specimens and biopsy specimens revealed only BE without dysplasia.

Table 3 Resection rates according to the final histologic result.

\begin{tabular}{|lcll|}
\hline & No. lesions & $\begin{array}{l}\text { En bloc } \\
\text { resection } \\
\text { rate, } \%\end{array}$ & $\begin{array}{l}\text { Complete } \\
\text { resection } \\
\text { rate, } \%\end{array}$ \\
\hline Low grade dysplasia & 3 & 100 & 100 \\
\hline High grade dysplasia & 8 & 100 & 100 \\
\hline EAC T1 $3^{1}$ & 11 & 73 & 64 \\
\hline EAC T1sm 11 & 6 & 67 & 50 \\
\hline EAC $\geq T 1 \mathrm{sm} 2$ & 5 & 80 & 0 \\
\hline SCC & 1 & 100 & 0 \\
\hline Total & 34 & 82 & 62 \\
\hline
\end{tabular}

EAC, esophageal adenocarcinoma; SCC, squamous cell carcinoma.

${ }^{1}$ At least (possible invasion of deeper structures). 


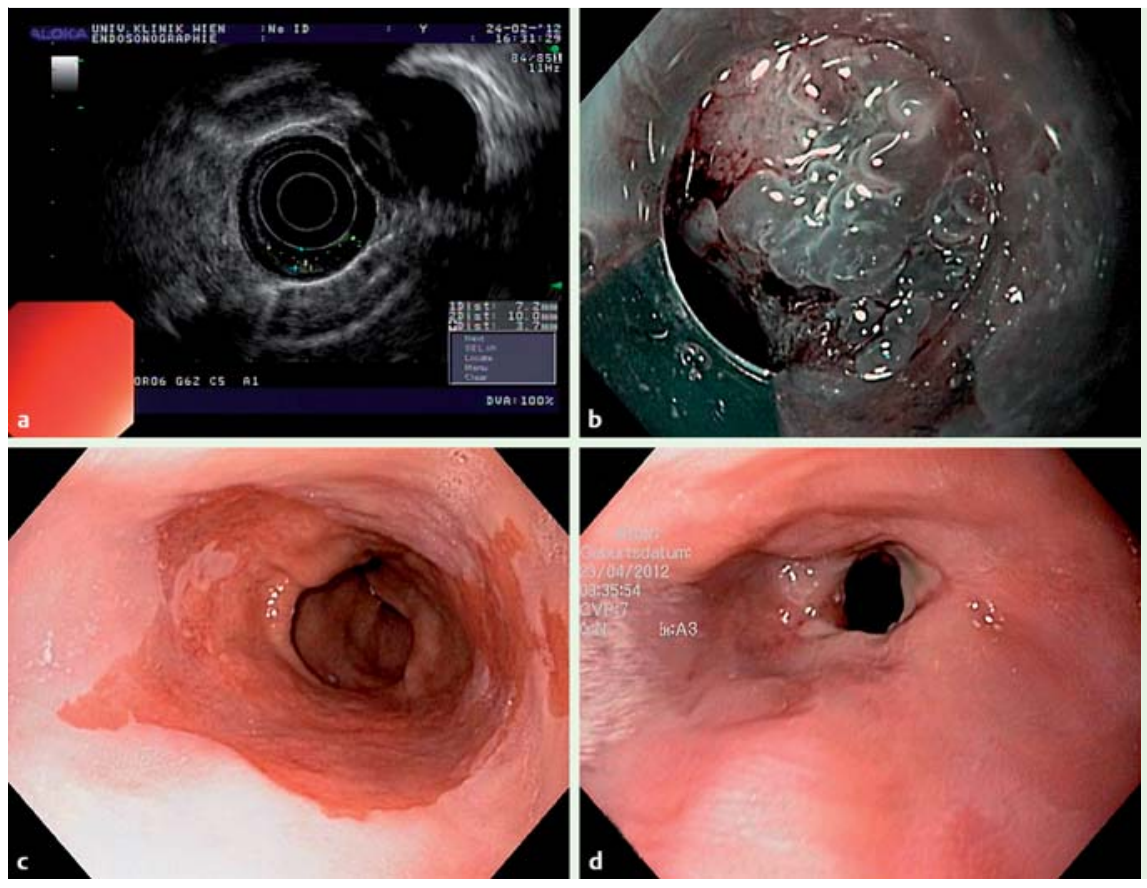

Fig. 2 Staging, mapping, and follow-up. a Endosonography showing tumor invasion of the submucosal layer. b Irregular surface and vessel structure in an adenocarcinoma with subsquamous tumor extension on narrow-band imaging.

c Barrett's esophagus without detectable lesions. d Esophageal stricture after endoscopic resection.
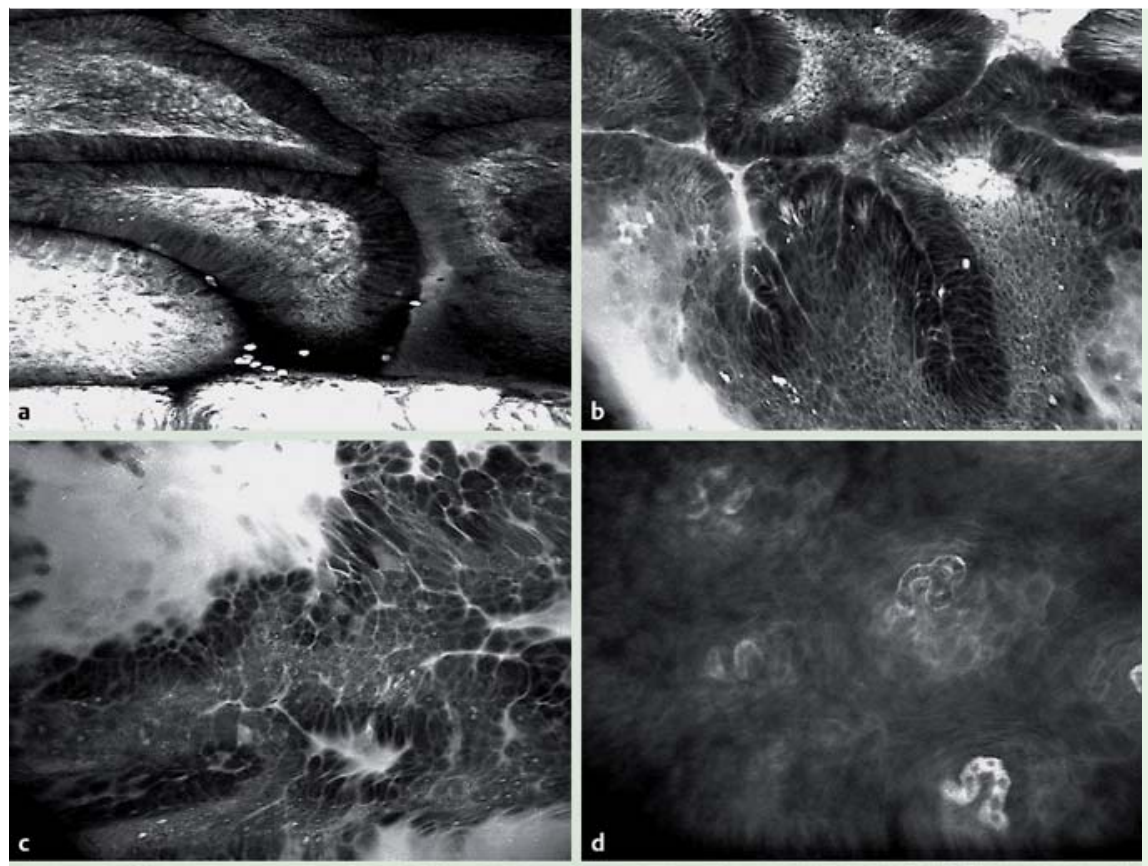

Fig. 3 Confocal laser endomicroscopy. a Nondysplastic Barrett's esophagus. b Barrett's esophagus with high grade dysplasia. c Esophageal adenocarcinoma. d Nondysplastic squamous epithelium. e Subsquamous extension of esophageal adenocarcinoma. $\mathbf{f}$ Squamous cell carcinoma.
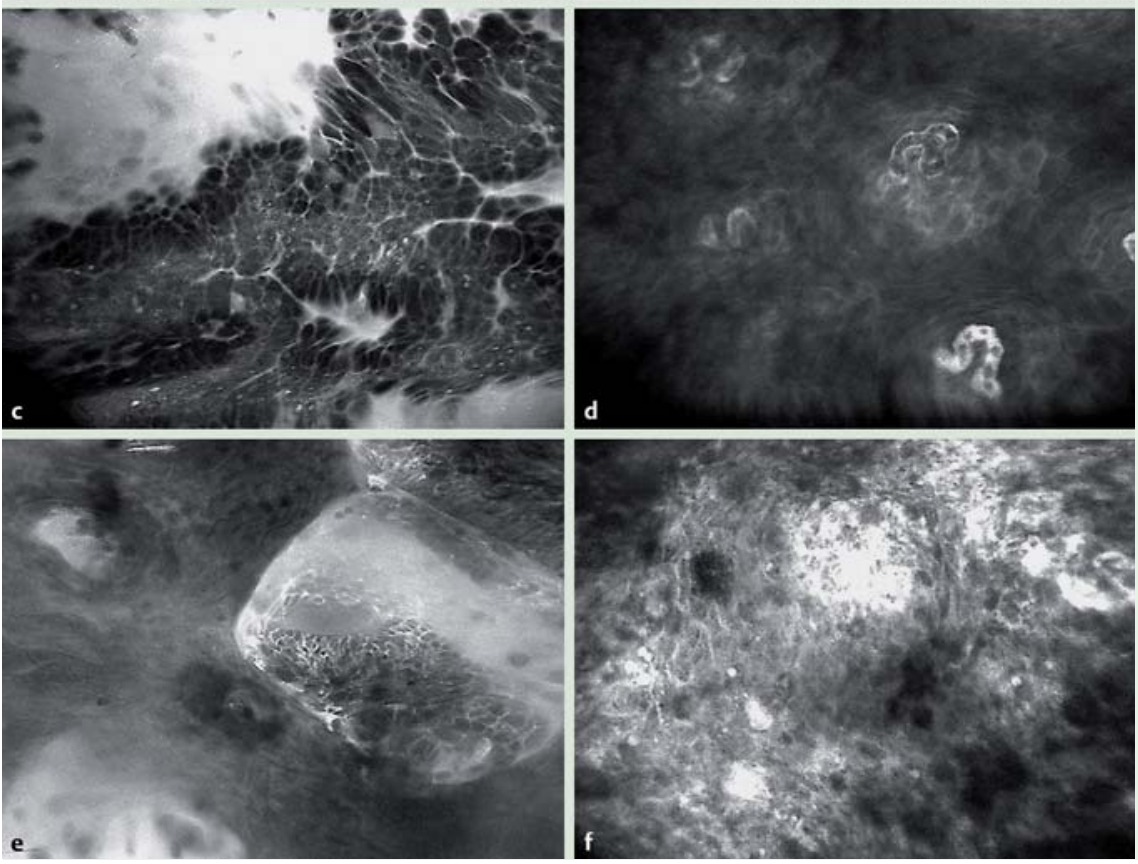


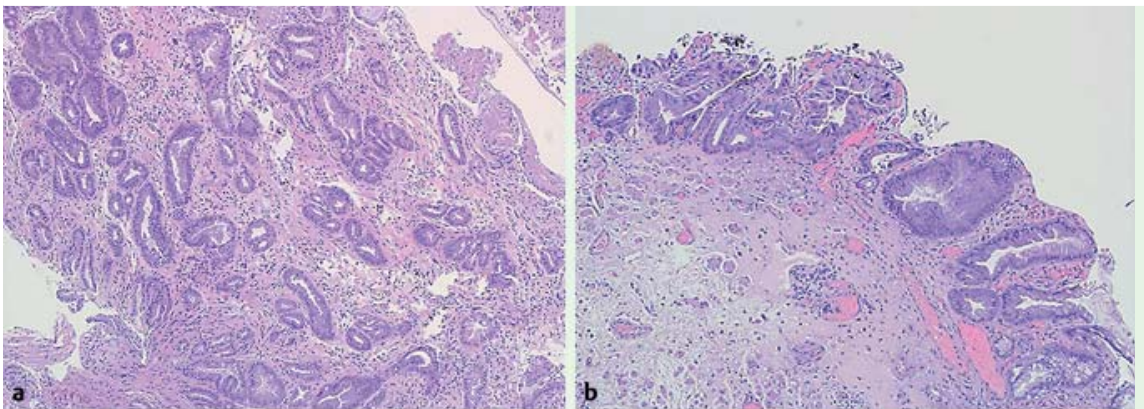

Fig. 4 Hematoxylin and eosin staining of histologic sections after endoscopic resection. a Nondysplastic Barrett's esophagus. b Barrett's esophagus with high grade dysplasia. c Esophageal adenocarcinoma (pT1m3). d Subsquamous extension of esophageal adenocarcinoma. $\mathbf{e}$ Detail of image in $\mathbf{d}$.
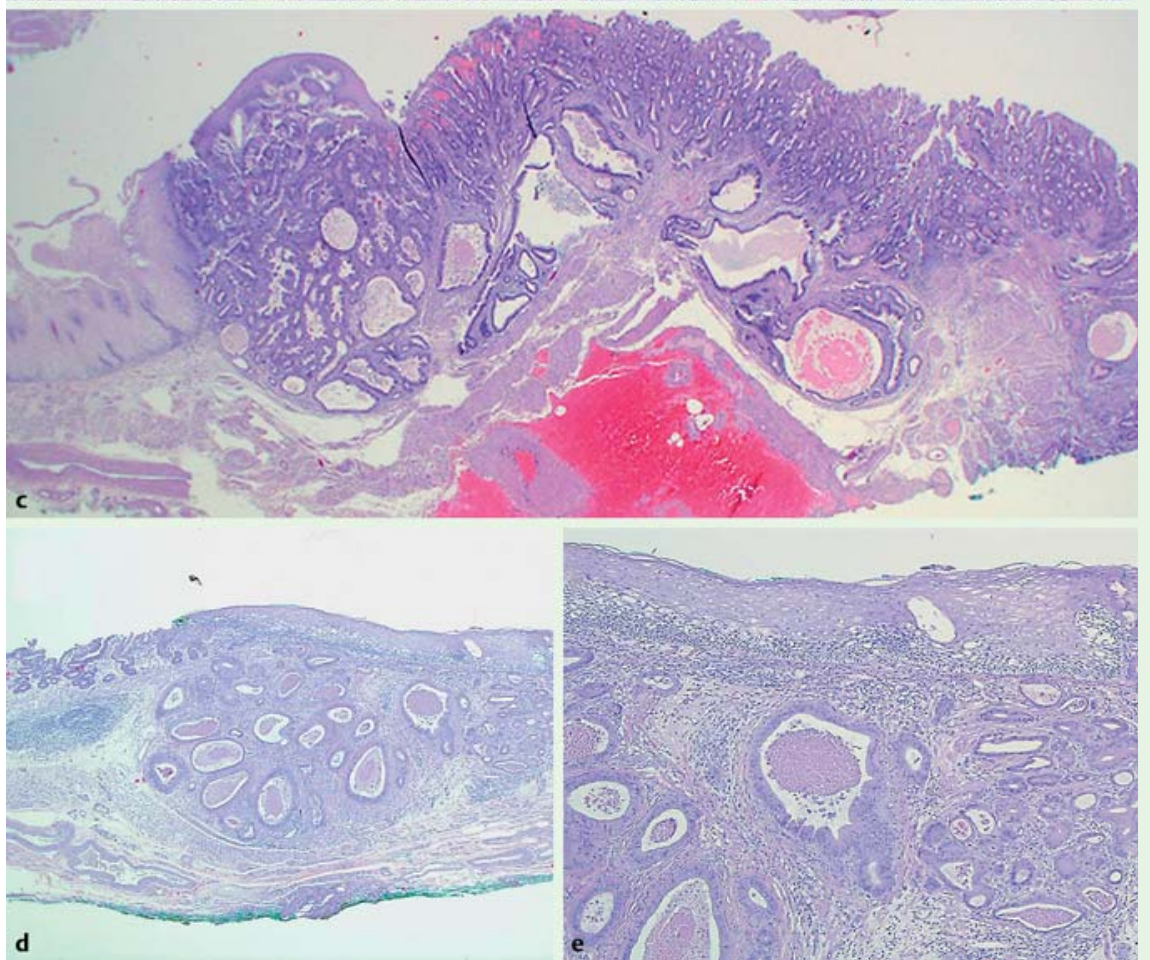

\section{Post-interventional follow-up}

After the endoscopic intervention, 12 (32\%) of the 38 study patients reported moderate retrosternal pain, which could be managed with analgesics. The median post-interventional hospital stay was 1 day (range, $1-3$ ). Hematemesis occurred on day 14 and day 16 after en bloc EMR and ESD, respectively, in 2 patients. In both patients, re-endoscopy revealed an ulcer with fibrin coverage at the site of the previous endoscopic resection but no active bleeding. In the context of this study, no early or delayed perforation was noticed. Of the 38 patients, 5 (13\%) reported difficulty swallowing solid food at the first clinical assessment within 3 weeks after the endoscopic intervention. In 1 patient, this symptom persisted at 4 weeks after the intervention. Re-endoscopy revealed a stricture at the esophagogastric junction (where EMR had been performed almost circularly for HGD; $\bullet$ Fig. 2d). This stricture was treated successfully during a single session of dilation with Savary-Gilliard bougies.

According to the final histologic results, 15 of the 38 study patients were considered to be at high risk for recurrence or metastatic disease. After individualized risk-benefit assessments, 9 of these patients (including the patient with the nonlifting sign during ESD) immediately underwent additional surgical therapy. The other 6 patients at high risk were followed endoscopically, along with the 23 patients in whom histology had revealed a low risk situation ( $\boldsymbol{\bullet}$ Fig. $\mathbf{1}$ ). During this follow-up, 12 patients with residual nondysplastic BE after endoscopic resection underwent a median number of 2 RFA sessions (range, $1-3$ ) for BE eradication. Advanced neoplasia was diagnosed at endoscopic follow-up in 4 patients in the high risk group and 2 patients in the low risk group ( 3 with HGD after 2, 14, and 16 months and 3 with EAC after 5, 8, and 8 months, respectively). Consequently, 2 patients of the high risk group underwent surgery (because of EAC with lymphatic vessel and deep submucosal invasion, respectively). The other patients underwent successful endoscopic treatment. After a median follow-up time of 13 months (range, 630 ), 24 patients were still being followed endoscopically ( 2 patients had died because of a nonrelated reason after 25 and 26 months, respectively, and 1 patient was lost to follow-up after 6 months).

\section{Discussion}

$\nabla$

This is the first prospective study that systematically used CLE to investigate lateral and subsquamous tumor extension in BE-associated neoplasia. This diagnostic approach, including the intravenous administration of fluorescein, was well tolerated and distinguished "neoplasia" from "no neoplasia" in almost all cases, as proved by the histologic analysis of resected specimens and conventional biopsy specimens. Compared with the macroscopic ap- 
pearance of lesions on high definition, white light endoscopy and NBI, the CLE findings provided an incremental yield in 7 (18\%) of 38 patients. Nevertheless, CLE failed to identify HGD in 4 patients without macroscopic lesions within $\mathrm{BE}$. This finding is consistent with recent publications showing only moderate sensitivity rates for cancer surveillance by CLE in patients with BE (in contrast to the initial studies already mentioned) $[15,16]$. If one considers that the field of view provided by CLE is $5 \times 5 \mu \mathrm{m}$, this may not be a surprising finding.

In this study, SST extension was detected before endoscopic resection in $6(16 \%)$ of 38 patients. Half of them were identified only by CLE ( $\bullet$ Fig. 3e) and later confirmed by conventional histology ( $\bullet$ Fig. 4d,e). According to the literature, attempts have been made to improve the visualization of SST extension by using chromoendoscopy with acetic acid and magnifying endoscopy with NBI $[17,18]$. However, systematic studies of SST extension are still lacking, although it has been reported to occur frequently in BE-associated neoplasia, admittedly affecting only a few millimeters in most cases. Concerning its clinical relevance, it should be stated that the detection of SST extension by CLE led to a change in the resection technique for 2 patients in this study (ESD instead of EMR). Based on the histologic assessment of these 2 patients, it must be assumed that without prior CLE, the extension of these lesions would have been underestimated, resulting in partial endoscopic resection.

In general, the endoscopic resection of BE-associated neoplasia proved to be safe and effective in this study, as shown by the good long-term follow-up results. The en bloc resection rate was good (82\%) for all underlying histologic types ( Table 3 ). This rate compares well with those in previous publications on the endoscopic resection of BE-associated neoplasia, which have reported en bloc resection rates of approximately $60 \%$ to $100 \%$ [19-21]. On the other hand, the complete resection rate was only moderate in this study, which must be attributed to the considerably high proportion of patients with EAC involving the submucosal layer. This finding could not be anticipated by EUS staging, which identified only 3 (27\%) of 11 cases of EAC with histologically proven submucosal invasion. Similar results have been reported in previous studies $[22,23]$.

Although CLE in itself is not a red flag technique, its use allowed us to identify 2 additional lesions during the systematic examination of additional BE segments and the surrounding squamous epithelium. However, these findings may be attributed to chance rather than to the specific diagnostic features of CLE because a conventional biopsy protocol might also have detected the lesions. Nevertheless, the cases nicely demonstrate that the onsite histologic feedback provided by CLE can be integrated into the planning for immediate endoscopic resection, which may reduce the number of repeated interventions, especially in view of the high prevalence of metachronous neoplasia in dysplastic BE [24].

The uncontrolled design and limited sample size may be considered shortcomings of this study. Although high definition, white light endoscopy with NBI and acetic acid chromoendoscopy were used in addition to CLE, the study was not designed to be a comparison trial of these modalities. Consequently, the endoscopist performing the CLE mapping was only partially blinded to the results of the earlier high definition, white light endoscopy, which should serve as the red flag technique for CLE. In fact, it was considered inappropriate to use only the standard definition endoscopy of the scope-based CLE system for targeting neoplastic lesions within BE. Of course, this issue could have been overcome by the use of a probe-based CLE system, which was not yet available during the planning of this study. On the other hand, the variable depth of tissue penetration offered by the scope-based CLE system proved to be essential for identifying SST extension underneath normal-looking CLE sections of superficial squamous epithelium (a feature not provided by probe-based CLE).

In summary, CLE served as a powerful supporting diagnostic tool for planning endoscopic resection by assessing lateral and subsquamous tumor extension of BE-associated neoplasia. Endoscopic treatment proved to be safe and effective in this study. In vivo imaging with CLE appears to be a pioneering technology for optimizing the current endoscopic management of BE-associated neoplasia and should be further investigated in randomized, controlled clinical trials.

\section{Competing interests: None}

\section{Institutions}

${ }^{1}$ Division of Gastroenterology and Hepatology, Department of Internal Medicine III, Medical University of Vienna, and

Gastroesophageal Tumor Unit, Comprehensive Cancer Center, Vienna, Austria

${ }^{2}$ Clinical Institute of Pathology, Medical University of Vienna, and Gastroesophageal Tumor Unit, Comprehensive Cancer Center, Vienna, Austria

${ }^{3}$ Department of Surgery, Medical University of Vienna, and Gastroesophageal Tumor Unit, Comprehensive Cancer Center, Vienna, Austria

${ }^{4}$ Division of Oncology, Department of Internal Medicine I, Medical University of Vienna, and Gastroesophageal Tumor Unit, Comprehensive Cancer Center, Vienna, Austria

\section{References}

1 Blot WJ, Devesa SS, Kneller RW et al. Rising incidence of adenocarcinoma of the esophagus and gastric cardia. JAMA 1991; 265: 1287-1289

2 Brown LM, Devesa SS. Epidemiologic trends in esophageal and gastric cancer in the United States. Surg Oncol Clin N Am 2002; 11: 235 - 256

3 Murray L, Watson P, Johnston B. Risk of adenocarcinoma in Barrett's oesophagus: population based study. BMJ 2003; 327: 534-535

4 Hvid-Jensen F, Pedersen L, Drewes AM et al. Incidence of adenocarcinoma among patients with Barrett's esophagus. N Engl J Med 2011; 365: $1375-1383$

5 Dolak $W$. The evil lies below the surface: subsquamous tumor extension in Barrett's adenocarcinoma. Endosc Int Open 2014; 02: E41 - E42

6 Anders M, Lucks Y, El-Masry MA et al. Subsquamous extension of intestinal metaplasia is detected in $98 \%$ of cases of neoplastic Barrett's esophagus. Clin Gastroenterol Hepatol 2014; 12: $405-410$

7 Nagami Y, Machida H, Shiba $M$ et al. Clinical efficacy of endoscopic submucosal dissection for adenocarcinomas of the esophagogastric junction. Endosc Int Open 2014; 02: E15 - E20

8 Polglase AL, McLaren WJ, Skinner SA et al. A fluorescence confocal endomicroscope for in vivo microscopy of the upper- and the lower-GI tract. Gastrointest Endosc 2005; 62: 686-695

9 Kiesslich R, Gossner L, Goetz M et al. In vivo histology of Barrett's esophagus and associated neoplasia by confocal laser endomicroscopy. Clin Gastroenterol Hepatol 2006; 4: 979-987

10 Dunbar KB, Okolo P3rd, Montgomery E. Confocal laser endomicroscopy in Barrett's esophagus and endoscopically inapparent Barrett's neoplasia: a prospective, randomized, double-blind, controlled, crossover trial. Gastrointest Endosc 2009; 70: 645-654

11 Levine DS, Blount PL, Rudolph RE et al. Safety of a systematic endoscopic biopsy protocol in patients with Barrett's esophagus. Am J Gastroenterol 2000; 95: 1152-1157

12 The Paris endoscopic classification of superficial neoplastic lesions: esophagus, stomach, and colon: November 30 to December 1, 2002. Gastrointest Endosc 2003; 58: S3 - S43

13 Edge SB, Byrd DR, Compton CC et al., eds. American Joint Committee on Cancer Staging Manual. 7th: ed. New York, NY: Springer; 2010: 103

14 Sharma P, Dent J, Armstrong $D$ et al. The development and validation of an endoscopic grading system for Barrett's esophagus: the Prague C \& M criteria. Gastroenterology 2006; 131: 1392 -1399

15 Bajbouj M, Vieth M, Rösch T et al. Probe-based confocal laser endomicroscopy compared with standard four-quadrant biopsy for evaluation of neoplasia in Barrett's esophagus. Endoscopy 2010; 42: 435-440 
16 Jayasekera C, Taylor AC, Desmond PV et al. Added value of narrow band imaging and confocal laser endomicroscopy in detecting Barrett's esophagus neoplasia. Endoscopy 2012; 44: 1089-1095

17 Yamagata T, Hirasawa D, Fujita $N$ et al. Efficacy of acetic acid-spraying method in diagnosing extension of Barrett's cancer under the squamous epithelium. Dig Endosc 2012; 24: 309-314

18 Omae M, Fujisaki J, Shimizu T et al. Magnifying endoscopy with narrow-band imaging findings in the diagnosis of Barrett's esophageal adenocarcinoma spreading below squamous epithelium. Dig Endosc 2013; 25: $162-167$

19 Vieth M, Ell C, Gossner L et al. Histological analysis of endoscopic resection specimens from 326 patients with Barrett's esophagus and early neoplasia. Endoscopy 2004; 36: 776 - 781

20 Neuhaus H, Terheggen G, Rutz EM et al. Endoscopic submucosal dissection plus radiofrequency ablation of neoplastic Barrett's esophagus. Endoscopy 2012; 44: 1105-1113
21 Nagami Y, Machida H, Shiba M et al. Clinical efficacy of endoscopic submucosal dissection for adenocarcinomas of the esophagogastric junction. Endosc Int Open 2014; 02: E15-E20

22 May A, Günter E, Roth $F$ et al. Accuracy of staging in early oesophageal cancer using high resolution endoscopy and high resolution endosonography: a comparative, prospective, and blinded trial. Gut 2004; 53: $634-640$

23 Larghi A, Lightdale CJ, Memeo L. EUS followed by EMR for staging of high-grade dysplasia and early cancer in Barrett's esophagus. Gastrointest Endosc 2005; 62: 16-23

24 Behrens A, May A, Gossner $L$ et al. Curative treatment for high-grade intraepithelial neoplasia in Barrett's esophagus. Endoscopy 2005; 37: 999-1005 